Original

\title{
The influence of polymerization conditions on color stability of three indirect composite materials
}

\author{
Hideyuki Imai ${ }^{1,2)}$, Hiroyasu Koizumi ${ }^{3,4)}$, Keiichi Sasaki²), and Hideo Matsumura ${ }^{3,4)}$ \\ 1)Dental Technician Training School, Nihon University School of Dentistry, Tokyo, Japan \\ 2)Division of Advanced Prosthetic Dentistry, Tohoku University Graduate School of Dentistry, Sendai, Japan \\ 3)Department of Fixed Prosthodontics, Nihon University School of Dentistry, Tokyo, Japan \\ 4)Division of Advanced Dental Treatment, Dental Research Center, Nihon University School of Dentistry, \\ Tokyo, Japan
}

(Received November 8, 2012; Accepted December 27, 2012)

\begin{abstract}
The aim of the present study was to evaluate color change and color stability against aqueous media of three indirect composite materials (Epricord, Estenia, and Twiny) that were polymerized with different systems. Disk-shaped specimens were prepared with their proprietary polymerization systems or with a metal halide light polymerization unit (Twinkle $\mathrm{X}$ ). The specimens were then immersed in water or tea. Change in color from baseline $(24$ h) to 4 weeks was determined with a chromameter (ShadeEye NCC) using a white background. The $L^{*}$, $a^{*}$, and $b^{*}$ values for both states were determined, and $\Delta \mathrm{E}^{*}{ }_{\mathrm{ab}}$ values were calculated. The Estenia specimens immersed in tea had significantly lower $\Delta L *(-7.0$ to -5.6) and significantly higher $\Delta E^{*}{ }_{a b}$ values (6.5 to 8.6) than did the Epricord and Twiny specimens, under all polymerization conditions. The results indicate that after tea immersion the Estenia material was less stable against color change than were the other two materials. The Twinkle $X$ metal halide unit was suitable for polymerization of the three composite materials, using an exposure period of $60 \mathrm{~s}$ or longer. (J Oral Sci 55, 51-55, 2013)
\end{abstract}

Keywords: color; composite; heat; light; polymerization.

Correspondence to Hideyuki Imai, Dental Technician Training School, Nihon University School of Dentistry, 1-8-13 KandaSurugadai, Chiyoda-ku, Tokyo 101-8310, Japan

Fax:+81-3-3219-8320 E-mail: imai.hideyuki@nihon-u.ac.jp

\section{Introduction}

Use of tooth-colored composite materials for restorations and partial denture veneers has increased substantially, probably due to improvements in the properties of the materials. However, several concerns have been reported. One such concern is color stability. A number of studies have reported changes in the color of composites (1-10). In addition, the polymeric matrix in composite materials is stained by beverages, food, and drugs (11-15).

A high-intensity light-polymerization apparatus has been developed (16), and use of this apparatus in combination with metal halide light sources was found to considerably improve the mechanical properties (17) and wear resistance (18) of composite materials. Although the stability of restorative materials against color change has been extensively studied, there is limited information on the color stability of indirect composite materials polymerized with different devices (19). The aim of the current project was to evaluate the influence of different varying polymerization conditions on the color stability of three indirect composite materials.

\section{Materials and Methods}

Indirect composite materials and polymerization apparatus

Three indirect composite materials designed for restoration and partial denture veneers were assessed (Table 1). Epricord (Kuraray Medical Inc., Tokyo, Japan) is composed of urethane tetramethacrylate (UTMA), triethyleneglycol dimethacrylate (TEGDMA), and $76.0 \mathrm{wt} \%$ filler. Estenia C\&B (Kuraray Medical Inc.) is composed 
Table 1 Indirect composite materials assessed

\begin{tabular}{llcl}
\hline Trade name & Manufacturer & Lot number & Composition (wt $\%)$ \\
\hline Epricord E2 & Kuraray Medical Inc., & 0065CA & UTMA, TEGDMA, $d l$-camphorquinone, pigment, 76.0\% filler (prepolymerized \\
& Tokyo, Japan & 0066AA & organic filler, silanated glass filler, silanated colloidal silica), others \\
Estenia C\&B E2 & Kuraray Medical Inc. & 0037BA & UTMA, methacrylate, $d l$-camphorquinone, pigment, 87.9\% filler (surface-treated \\
& & $0037 \mathrm{BB}$ & alumina microfiller, silanated glass ceramic filler), others \\
\multirow{2}{*}{ Twiny E2 } & Yamamoto Precious Metal & 1031128 & UTMA, TEGDMA, 79.0\% filler, pigment, others \\
& Co., Ltd., Osaka, Japan & & \\
\hline
\end{tabular}

UTMA, urethane tetramethacrylate; TEGDMA, triethyleneglycol dimethacrylate

Table 2 Polymerization apparatus employed

\begin{tabular}{llll}
\hline Trade name & Manufacturer & Source & Polymerization condition \\
\hline$\alpha$-Light II & J. Morita Corp., Suita, Japan & Halogen lamp: $360 \mathrm{~W} \times 1,400-600 \mathrm{~nm} * ;$ & $180 \mathrm{~s}$ \\
& & Fluorescent tubes: $27 \mathrm{~W} \times 2,400-550 \mathrm{~nm} *$ & \\
KL-310 & J. Morita Corp. & Heat oven & $110^{\circ} \mathrm{C}, 15 \mathrm{~min}$ \\
Twinkle X & Toho Dental Products, Saitama, Japan & Metal halide lamps, $150 \mathrm{~W} \times 2,250-600 \mathrm{~nm} * *$ & $30 \mathrm{~s}, 60 \mathrm{~s}$, and $90 \mathrm{~s}$ \\
\hline
\end{tabular}

*Reference $18 ; * *$ Reference 16.

of UTMA, other methacrylate, and $87.9 \mathrm{wt} \%$ filler. Twiny (Yamamoto Precious Metal Co., Ltd., Osaka, Japan) is composed of UTMA, TEGDMA, and $79.0 \mathrm{wt} \%$ filler.

Three polymerization devices were used (Table 2). The $\alpha$-Light II (J. Morita Corp., Suita, Japan) unit is equipped with a halogen lamp and two fluorescent tubes (18). The KL-310 (J. Morita Corp.) apparatus is a box-type heat oven. The Twinkle X (Toho Dental Products, Saitama, Japan) unit is equipped with two high-intensity discharge metal halide lamps (16).

\section{Specimen preparation}

The composite materials were filled into steel molds (15 $\mathrm{mm}$ in diameter by $2 \mathrm{~mm}$ in height) placed on a glass plate (thickness, $1.3 \mathrm{~mm}$; Micro Slide Glass, Matsunami Glass Ind., Ltd., Osaka, Japan). The composite material was filled into the mold, and the surface was covered with the 1.3-mm glass plate. The specimen was then polymerized with its proprietary system or with the Twinkle $X$ unit for 30-90 s (Table 2). The proprietary system for the Epricord material was light exposed for $180 \mathrm{~s}$ with the $\alpha$-Light II unit, and the polymerization system for the Estenia C\&B and Twiny materials was light exposed with the $\alpha$-Light II unit for $180 \mathrm{~s}$, followed by heating with the KL-310 oven for $15 \mathrm{~min}$ at $110^{\circ} \mathrm{C}$. After polymerization, both surfaces of each specimen were ground with silicon-carbide ( $\mathrm{SiC}$ ) abrasive papers (\#800-\#2,000, WetorDry Tri-M-ite, 3M Corp., St. Paul, MN, USA) under running water. The ground specimens were then polished with felt (TexMet 1500, Buehler Ltd., Lake Bluff, IL, USA) and diamond slurry (3, 1, and 0.25 $\mu \mathrm{m}$, MetaDi, Buehler Ltd.). The specimen thickness was adjusted to $1.0 \mathrm{~mm}$ using a digital caliper (Mitutoyo Corp., Kawasaki, Japan). The prepared specimens were then placed in an incubator at $37^{\circ} \mathrm{C}$ for $24 \mathrm{~h}$ in a dry state.

\section{Color determination and calculation of color difference}

After 24-h storage, specimens were immersed in purified water or tea for 4 weeks (28 days). The tea solution was prepared by placing three tea bags (15 g; Day \& Day Teabags, Mitsui Norin Co., Ltd., Tokyo, Japan) in 1.0 L of boiling water and brewing for $5 \mathrm{~min}$ (19). The color values $\left(\mathrm{L}^{*}, \mathrm{a}^{*}\right.$, and $\left.\mathrm{b}^{*}\right)$ of specimens were determined with a chromameter (ShadeEye NCC, Shofu Inc., Kyoto, Japan) using a white background (Konica Minolta Holdings Inc., Osaka, Japan). Mean color values of the white background were $\mathrm{L}^{*}=97.3, \mathrm{a}^{*}=0.1$, and $\mathrm{b}^{*}=-1.3$.

The color of the dry-state specimens was measured $24 \mathrm{~h}$ after preparation, without immersion in solution. Immersed specimens (4 weeks) were rinsed with water and dried with absorbent paper. Color was measured according to the Commission Internationale de 1'Eclairage (CIE) 1976 L*a*b* color space system relative to the standard illuminant $\mathrm{D}_{65}$ and observer functions ( $2^{\circ}$ visual field). The system was calibrated at the start of each measurement with a white calibration tile provided by the manufacturer. The aperture of the chromameter was placed on the specimen surface. Color measurements were performed six times around the center of each specimen, and mean values were calculated. Nine specimens were tested for each material and condition. The color difference between pre- and post-immersion values $\left(\Delta \mathrm{E}^{*}{ }_{\mathrm{ab}}\right)$ was determined for each specimen. The following equation was used to calculate $\Delta \mathrm{E}^{*}$ ab values: $\Delta \mathrm{E}_{\mathrm{ab}}^{*}=\left\{\left(\Delta \mathrm{L}^{*}\right)^{2}+\left(\Delta \mathrm{a}^{*}\right)^{2}+\left(\Delta \mathrm{b}^{*}\right)^{2}\right\}^{1 / 2}$. 
Table $3 \Delta \mathrm{L}^{*}$ values for white background (4 weeks)

\begin{tabular}{|c|c|c|c|c|c|c|c|c|c|}
\hline \multirow[b]{2}{*}{ Polymerization mode } & \multicolumn{5}{|c|}{ Water } & \multicolumn{4}{|c|}{ Tea } \\
\hline & Estenia & & Epricord & Twiny & $\mathrm{K}-\mathrm{W}$ test & Estenia & Epricord & Twiny & $\mathrm{K}-\mathrm{W}$ test \\
\hline Proprietary system & $-0.8(0.4)^{*}$ & $\mathrm{ab}$ & $-0.3(1.2)^{* *}$ & a $0(0.3)^{* *}$ & ab Significant & $-6.3(0.4) * a b$ & $-3.0(1.1)^{* *}$ & $-2.0(0.5)^{* *} \mathrm{a}$ & Significant \\
\hline Twinkle X $30 \mathrm{~s}$ & $-1.2(0.4)^{*}, * *$ & $\mathrm{a}$ & $-1.6(0.7)^{*}$ & $\mathrm{~b}-0.8(0.4)^{* *}$ & a Significant & $-7.0(0.4)^{*} \mathrm{a}$ & $-2.7(0.5)^{* *}$ & $-4.8(1.5)^{* *} b$ & Significant \\
\hline Twinkle X $60 \mathrm{~s}$ & $-0.6(0.6)^{*}$ & $\mathrm{ab}$ & $-0.8(0.8)^{*}$ & ab $0.1(0.8)^{*}$ & Not significant & $-6.0(0.9)^{*} \mathrm{ab}$ & $-2.7(1.7)^{* *}$ & $-3.0(0.9)^{* *} \mathrm{ab}$ & b Significant \\
\hline Twinkle X $90 \mathrm{~s}$ & $-0.5(0.3)^{*}$ & $\mathrm{~b}$ & $-0.6(1.0)^{*}$ & $\mathrm{ab} 0.2(0.5)^{* *}$ & Significant & $-5.6(0.7) * b$ & $-2.8(0.8)^{* *}$ & $-2.9(0.6)^{* *} \mathrm{ab}$ & b Significant \\
\hline
\end{tabular}

$n=9$. Means (SD). Identical letters indicate absence of a significant difference among polymerization modes (Dunn's test, $P>0.05$ ).

$\mathrm{K}-\mathrm{W}$ test, Kruskal-Wallis test. K-W test and Dunn's test, difference between Estenia, Epricord, and Twiny (significant; $P<0.05$ ). Identical symbols indicate absence of a significant difference.

Table $4 \Delta \mathrm{a}^{*}$ values for white background (4 weeks)

\begin{tabular}{|c|c|c|c|c|c|c|c|c|c|c|c|c|c|}
\hline \multirow[b]{2}{*}{ Polymerization mode } & \multicolumn{7}{|c|}{ Water } & \multicolumn{6}{|c|}{ Tea } \\
\hline & Estenia & & Epricord & & Twiny & & $\mathrm{K}-\mathrm{W}$ test & Estenia & & Epricord & & Twiny & $\mathrm{K}-\mathrm{W}$ test \\
\hline Proprietary system & $-0.1(0.3)^{*}$ & $\mathrm{a}$ & $0(0.2)^{*}$ & $\mathrm{a}$ & $0(0.1)^{*}$ & $\mathrm{a}$ & Not significant & $1.6(0.2)^{*}$ & $\mathrm{a}$ & $1.2(0.2) * * *$ & $\mathrm{a}$ & $0.6(0.2)^{* *} \mathrm{a}$ & Significant \\
\hline Twinkle X $30 \mathrm{~s}$ & $0.7(0.1)^{*}$ & $\mathrm{bc}$ & $-0.5(0.3)^{* *}$ & $\mathrm{~b}$ & $0.5(0.1)^{* * *}$ & bc & Significant & $2.3(0.2)^{*}$ & $\mathrm{~b}$ & $1.0(0.3)^{* *}$ & $\mathrm{ac}$ & $1.2(0.4)^{* *} \mathrm{~b}$ & Significant \\
\hline Twinkle X $60 \mathrm{~s}$ & $0.6(0.1)^{*}$ & $\mathrm{ac}$ & $-0.1(0.2)^{* *}$ & ac & $0.2(0.1)^{* *}$ & $\mathrm{ac}$ & Significant & $2.0(0.2)^{*}$ & $\mathrm{bc}$ & $0.5(0.5)^{* *}$ & $\mathrm{bc}$ & $0.9(0.2)^{* *} \mathrm{ab}$ & Significant \\
\hline Twinkle X $90 \mathrm{~s}$ & $0.7(0.1)^{*}$ & $\mathrm{bc}$ & $-0.4(0.2)^{* *}$ & $\mathrm{bc}$ & $0.2(0.1)^{* * *}$ & $\mathrm{a}$ & Significant & $1.9(0.2)^{*}$ & $\mathrm{ac}$ & $0.4(0.3)^{* *}$ & $\mathrm{~b}$ & $0.7(0.3)^{* *} \mathrm{a}$ & Significant \\
\hline
\end{tabular}

$n=9$. Means (SD). Identical letters indicate absence of a significant difference among polymerization modes (Dunn's test, $P>0.05$ ).

$\mathrm{K}-\mathrm{W}$ test, Kruskal-Wallis test. K-W test and Dunn's test, difference between Estenia, Epricord, and Twiny (significant; $P<0.05$ ). Identical symbols indicate absence of a significant difference.

Table $5 \Delta \mathrm{b}^{*}$ values for white background (4 weeks)

\begin{tabular}{|c|c|c|c|c|c|c|c|c|c|c|c|c|c|}
\hline \multirow[b]{2}{*}{ Polymerization mode } & \multicolumn{7}{|c|}{ Water } & \multicolumn{6}{|c|}{ Tea } \\
\hline & Estenia & & Epricord & & Twiny & & $\mathrm{K}-\mathrm{W}$ test & Estenia & Epricord & & Twiny & & $\mathrm{K}-\mathrm{W}$ test \\
\hline Proprietary system & $-1.0(0.2)^{*}$ & $\mathrm{a}$ & $1.1(0.3)^{* *}$ & $\mathrm{ab}$ & $-1.1(0.2)^{*}$ & $\mathrm{a}$ & Significant & $3.8(0.4)^{*} \mathrm{ab}$ & $1.2(0.3)^{* *}$ & $\mathrm{a}$ & $-0.6(0.2)^{* * *}$ & $\mathrm{a}$ & Significant \\
\hline Twinkle X $30 \mathrm{~s}$ & $-0.3(0.2)^{*}$ & $\mathrm{~b}$ & $-1.4(0.1)^{* *}$ & $\mathrm{~b}$ & $-2.5(0.1)^{* * *}$ & $\mathrm{~b}$ & Significant & $4.4(0.4)^{*} \quad \mathrm{a}$ & $1.3(0.2)^{* *}$ & $\mathrm{a}$ & $1.9(2.0)^{* *}$ & $\mathrm{~b}$ & Significant \\
\hline Twinkle X $60 \mathrm{~s}$ & $-0.5(0.2)^{*}$ & $\mathrm{~b}$ & $1.4(0.3)^{* *}$ & $\mathrm{ac}$ & $-1.2(0.2)^{* * *}$ & $\mathrm{a}$ & Significant & $3.1(0.4)^{*} \quad \mathrm{~b}$ & $1.7(0.4)^{* *}$ & $\mathrm{ac}$ & $-0.7(0.6)^{* *}$ & $\mathrm{a}$ & Significant \\
\hline Twinkle X $90 \mathrm{~s}$ & $-1.0(0.1)^{*}$ & $\mathrm{a}$ & $2.2(0.3)^{* *}$ & $\mathrm{c}$ & $-0.7(0.3)^{*}$ & $\mathrm{a}$ & Significant & $2.7(0.4)^{*} \mathrm{bc}$ & $2.8(0.2)^{*}$ & $\mathrm{bc}$ & $0.2(0.7)^{* *}$ & $\mathrm{ab}$ & Significant \\
\hline
\end{tabular}

$n=9$. Means (SD). Identical letters indicate absence of a significant difference among polymerization modes (Dunn's test, $P>0.05$ ).

$\mathrm{K}-\mathrm{W}$ test, Kruskal-Wallis test. K-W test and Dunn's test, difference between Estenia, Epricord, and Twiny (significant; $P<0.05$ ). Identical symbols indicate absence of a significant difference.

Table $6 \Delta \mathrm{E}^{*}{ }_{\mathrm{ab}}$ values for white background (4 weeks)

\begin{tabular}{|c|c|c|c|c|c|c|c|c|c|c|c|c|}
\hline \multirow[b]{2}{*}{ Polymerization mode } & \multicolumn{6}{|c|}{ Water } & \multicolumn{6}{|c|}{ Tea } \\
\hline & Estenia & Epricord & & Twiny & & $\mathrm{K}-\mathrm{W}$ test & Estenia & Epricord & & Twiny & & $\mathrm{K}-\mathrm{W}$ test \\
\hline Proprietary system & $1.4(0.4) * a b$ & $1.5(0.7)^{*}$ & $\mathrm{a}$ & $1.2(0.3)^{*}$ & $\mathrm{a}$ & Not significant & $7.6(0.5) * a b$ & $3.5(0.8)^{* *}$ & $\mathrm{a}$ & $2.2(0.5)^{* *}$ & $\mathrm{a}$ & Significant \\
\hline Twinkle X $30 \mathrm{~s}$ & $1.5(0.3)^{*} \quad \mathrm{a}$ & $2.3(0.5)^{* *}$ & $a b$ & $2.7(0.2)^{* *}$ & $\mathrm{bc}$ & Significant & $8.6(0.4)^{*} \quad \mathrm{a}$ & $3.2(0.5)^{* *}$ & $\mathrm{a}$ & $5.6(1.6)^{* *}$ & $\mathrm{~b}$ & Significant \\
\hline Twinkle X $60 \mathrm{~s}$ & $1.1(0.3)^{*} \quad b$ & $1.8(0.5)^{* *}$ & $\mathrm{ab}$ & $1.4(0.3)^{* * * *}$ & $\mathrm{ac}$ & Significant & $7.1(0.7)^{*}$ & $3.4(1.4)^{* *}$ & $\mathrm{a}$ & $3.3(0.9)^{* *}$ & $\mathrm{ab}$ & Significant \\
\hline Twinkle X $90 \mathrm{~s}$ & $1.4(0.1)^{*} \mathrm{ab}$ & $2.5(0.4)^{* *}$ & $\mathrm{~b}$ & $0.9(0.3)^{*}$ & $\mathrm{a}$ & Significant & $6.5(0.7)^{*}$ & $4.0(0.5)^{* *}$ & $\mathrm{a}$ & $3.1(0.6)^{* *}$ & $a b$ & Significant \\
\hline
\end{tabular}

$n=9$. Means (SD). Identical letters indicate absence of a significant difference among polymerization modes (Dunn's test, $P>0.05$ ).

K-W test, Kruskal-Wallis test. K-W test and Dunn's test, difference between Estenia, Epricord, and Twiny (significant; $P<0.05$ ). Identical symbols indicate absence of a significant difference.

\section{Statistical analysis}

The $\Delta \mathrm{L}^{*}, \Delta \mathrm{a}^{*}, \Delta \mathrm{b}^{*}$, and $\Delta \mathrm{E}^{*}{ }_{\text {ab }}$ values were analyzed by the Kolmogorov-Smirnov test for normality of distribution (SPSS 15.0, SPSS Inc., Chicago, IL, USA). When the results of the Kolmogorov-Smirnov test did not show normality of distribution, the Kruskal-Wallis test (SPSS 15.0) and Dunn's comparison test (GraphPad Prism 5, GraphPad Software Inc., La Jolla, CA, USA) were performed. The value for statistical significance was set at $\alpha=0.05$.

\section{Results}

The Kolmogorov-Smirnov test showed that some categories in several groups did not have normal distributions. The results were therefore analyzed with the KruskalWallis test and Dunn's comparison test. The mean (SD) of $\Delta \mathrm{L}^{*}, \Delta \mathrm{a}^{*}, \Delta \mathrm{b}^{*}$, and $\Delta \mathrm{E}^{*}{ }_{\text {ab }}$ are shown in Tables 3 through 6 .

Table 3 summarizes mean $\Delta \mathrm{L}^{*}$ values. The $\Delta \mathrm{L}^{*}$ of specimens immersed in water ranged from -1.2 to -0.5 for Estenia, -1.6 to -0.3 for Epricord, and -0.8 to 0.2 for Twiny. For specimens immersed in tea, $\Delta \mathrm{L}^{*}$ ranged from 
-7.0 to -5.6 for Estenia, -3.0 to -2.7 for Epricord, and -4.8 to -2.0 for Twiny. The Estenia specimens immersed in tea had a significantly lower $\Delta \mathrm{L}^{*}$ than the Epricord and Twiny specimens under all polymerization conditions.

Table 4 shows $\Delta \mathrm{a}^{*}$ values. The $\Delta \mathrm{a}^{*}$ of specimens immersed in water ranged from -0.1 to 0.7 for Estenia, -0.5 to 0 for Epricord, and 0 to 0.5 for Twiny. For specimens immersed in tea, $\Delta \mathrm{a}^{*}$ ranged from 1.6 to 2.3 for Estenia, 0.4 to 1.2 for Epricord, and 0.6 to 1.2 for Twiny.

Mean $\Delta \mathrm{b}^{*}$ values are shown in Table 5 . The $\Delta \mathrm{b}^{*}$ of specimens immersed in water ranged from -1.0 to -0.3 for Estenia, -1.4 to 2.2 for Epricord, and -2.5 to -0.7 for Twiny. For specimens immersed in tea, $\Delta \mathrm{b}^{*}$ ranged from 2.7 to 4.4 for Estenia, 1.2 to 2.8 for Epricord, and -0.7 to 1.9 for Twiny. The $\Delta \mathrm{b}^{*}$ of specimens polymerized with proprietary systems and immersed in tea was ranked in ascending order as Twiny (-0.6), Epricord (1.2), and Estenia (3.8), and the trend was statistically significant.

Mean $\Delta \mathrm{E}^{*}{ }_{\text {ab }}$ values are summarized in Table 6 . The $\Delta \mathrm{E}^{*}{ }_{\mathrm{ab}}$ of specimens immersed in water ranged from 1.1 to 1.5 for Estenia, 1.5 to 2.5 for Epricord, and 0.9 to 2.7 for Twiny. For specimens immersed in tea, $\Delta \mathrm{E}^{*}$ ab ranged from 6.5 to 8.6 for Estenia, 3.2 to 4.0 for Epricord, and 2.2 to 5.6 for Twiny. Estenia specimens immersed in tea had significantly higher $\Delta \mathrm{E}^{*}$ ab value than the Epricord and Twiny specimens for all polymerization conditions.

\section{Discussion}

This study evaluated color change after immersion in water or tea in three indirect composite materials polymerized with different systems. A number of storage media have been proposed, including coffee $(10-13,20,21)$, cola $(11,13)$, juice $(13,15)$, red wine $(11-13,20,21)$, soy sauce (15), and tea $(11,12,20,21)$. This study employed tea as a coloring solution because it is relatively homogeneous as a coloring solution and because the color of tea is stable during storage.

$L^{*}$ represents the brightness of materials. Table 3 shows that change in $\mathrm{L}^{*}$ did not significantly differ after immersion in water among the three materials. However, $\mathrm{L}^{*}$ was considerably lower after immersion in tea, especially for the Estenia material. The authors consider that the reduction in $\mathrm{L}^{*}$ after tea immersion was due to the coloring ingredients in tea. The results are in agreement with those of other studies that used tea as a storage medium $(11,12,19)$. Also, the greater reduction in $L^{*}$ for the Estenia material may be due to penetration of coloring ingredients into the interface between the matrix polymer and inorganic filler or into microvoids originally present in the Estenia material. The results shown in Table 1 show that the polymerization performance of the proprietary systems and the Twinkle X unit did not particularly differ and that light exposure of longer than $60 \mathrm{~s}$ was acceptable for all materials treated with the Twinkle $\mathrm{X}$ unit.

The $\Delta \mathrm{a}^{*}$ value of the three composite materials was not greatly affected by water storage and increased after storage in tea. In the CIE $1976 \mathrm{~L}^{*} \mathrm{a}^{*} \mathrm{~b}^{*}$ color space system, an increase in $\mathrm{a}^{*}$ represents increased red, and a decrease in $\mathrm{a}^{*}$ represents increased green. The results of the present study show that redness increased in all materials, especially for Estenia, after tea immersion, regardless of polymerization system. Similar results were seen in the $b^{*}$ values of the specimens, probably because yellowish color change was accelerated and bluish color change was suppressed by the original color of the tea solution.

According to the abovementioned equation, $\Delta \mathrm{E}_{\mathrm{ab}}^{*}$ represents total color change in specimens. The results for $\Delta \mathrm{E}^{*}$ ab (Table 6) indicate that light exposure for $30 \mathrm{~s}$ with the Twinkle $\mathrm{X}$ unit was insufficient for polymerization of Twiny. Also, Estenia was less stable against color change than were the other two materials after tea immersion, regardless of polymerization conditions. The results are likely due to the somewhat rougher surface characteristics of Estenia, which is a highly loaded composite material (18).

Dental technicians desire composite materials that can be simply and quickly polymerized. The Twinkle X unit, with a light exposure period greater than $60 \mathrm{~s}$, had better polymerization performance than the proprietary systems of the three investigated materials. Application of highintensity polymerization apparatus is recommended to improve stability against color change of indirect composite materials.

\section{Acknowledgments}

This work was supported in part by a Grant-in-Aid for Scientific Research (C) 23592871 (2011-2012) from the Japan Society for the Promotion of Science (JSPS) and by a Grant from the Dental Research Center (B) Nihon University School of Dentistry (2012). The experimental results of this article were submitted by one of the authors (H.I.) as a master's thesis in Dentistry (March 2012) to the Tohoku University Graduate School of Dentistry.

\section{References}

1. Mutlu-Sagesen L, Ergün G, Ozkan Y, Semiz M (2005) Color stability of a dental composite after immersion in various media. Dent Mater J 24, 382-390.

2. Arikawa H, Kanie T, Fujii K, Takahashi H, Ban S (2007) Effect of filler properties in composite resins on light transmittance characteristics and color. Dent Mater J 26, 38-44.

3. Fontes ST, Fernández MR, de Moura CM, Meireles SS (2009) Color stability of a nanofill composite: effect of different 
immersion media. J Appl Oral Sci 17, 388-391.

4. Cruvinel DR, Garcia Lda F, Consani S, de Carvalho Panzeri Pires-de-Souza F (2010) Composites associated with pulpprotection material: color-stability analysis after accelerated artificial aging. Eur J Dent 4, 6-11.

5. Mundim FM, Garcia Lda F, Pires-de-Souza Fde C (2010) Effect of staining solutions and repolishing on color stability of direct composites. J Appl Oral Sci 18, 249-254.

6. Papadopoulos T, Sarafianou A, Hatzikyriakos A (2010) Colour stability of veneering composites after accelerated aging. Eur J Dent 4, 137-142.

7. Lee YK, Yu B, Lim HN, Lim JI (2011) Difference in the color stability of direct and indirect resin composites. J Appl Oral Sci 19, 154-160.

8. Domingos PA, Garcia PP, de Oliveira AL, Palma-Dibb RG (2011) Composite resin color stability: influence of light sources and immersion media. J Appl Oral Sci 19, 204-211.

9. Güler AU, Duran I, Yücel AÇ, Ozkan P (2011) Effects of air-polishing powders on color stability of composite resins. J Appl Oral Sci 19, 505-510.

10. Schmitt VL, Puppin-Rontani RM, Naufel FS, Nahsan FP, Alexandre Coelho Sinhoreti M, Baseggio W (2011) Effect of the polishing procedures on color stability and surface roughness of composite resins. ISRN Dent 2011:617672.

11. Ertaş E, Güler AU, Yücel AC, Köprülü H, Güler E (2006) Color stability of resin composites after immersion in different drinks. Dent Mater J 25, 371-376.

12. Omata Y, Uno S, Nakaoki Y, Tanaka T, Sano H, Yoshida S et al. (2006) Staining of hybrid composites with coffee, oolong tea, or red wine. Dent Mater J 25, 125-131.

13. Topcu FT, Sahinkesen G, Yamanel K, Erdemir U, Oktay EA,
Ersahan S (2009) Influence of different drinks on the colour stability of dental resin composites. Eur J Dent 3, 50-56.

14. Galvão AP, Jacques LB, Dantas L, Mathias P, Mallmann A (2010) Effect of lipstick on composite resin color at different application times. J Appl Oral Sci 18, 566-571.

15. Soares-Geraldo D, Scaramucci T, Steagall-Jr W, Braga SR, Sobral MA (2011) Interaction between staining and degradation of a composite resin in contact with colored foods. Braz Oral Res 25, 369-375.

16. Matsumura H, Tanoue N, Atsuta M, Kitazawa S (1997) A metal halide light source for laboratory curing of prosthetic composite materials. J Dent Res 76, 688-693.

17. Satsukawa H, Koizumi H, Tanoue N, Nemoto M, Ogino T, Matsumura H (2005) Properties of an indirect composite material polymerized with two different laboratory polymerizing systems. Dent Mater J 24, 377-381.

18. Hirata M, Koizumi H, Tanoue N, Ogino T, Murakami M, Matsumura H (2011) Influence of laboratory light sources on the wear characteristics of indirect composites. Dent Mater J 30, 127-135.

19. Nakazawa M (2009) Color stability of indirect composite materials polymerized with different polymerization systems. J Oral Sci 51, 267-273.

20. Stawarczyk B, Egli R, Roos M, Ozcan M, Hämmerle CH (2011) The impact of in vitro aging on the mechanical and optical properties of indirect veneering composite resins. J Prosthet Dent 106, 386-398.

21. Gawriołek M, Sikorska E, Ferreira LF, Costa AI, Khmelinskii I, Krawczyk A et al. (2012) Color and luminescence stability of selected dental materials in vitro. J Prosthodont 21, 112-122. 